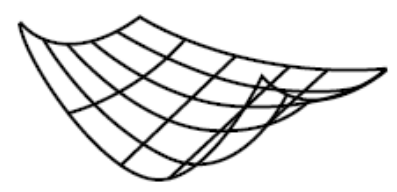

UNIVERSITY OF

TECHNOLOGY SYDNEY

QUANTITATIVE FINANCE

RESEARCH CENTRE

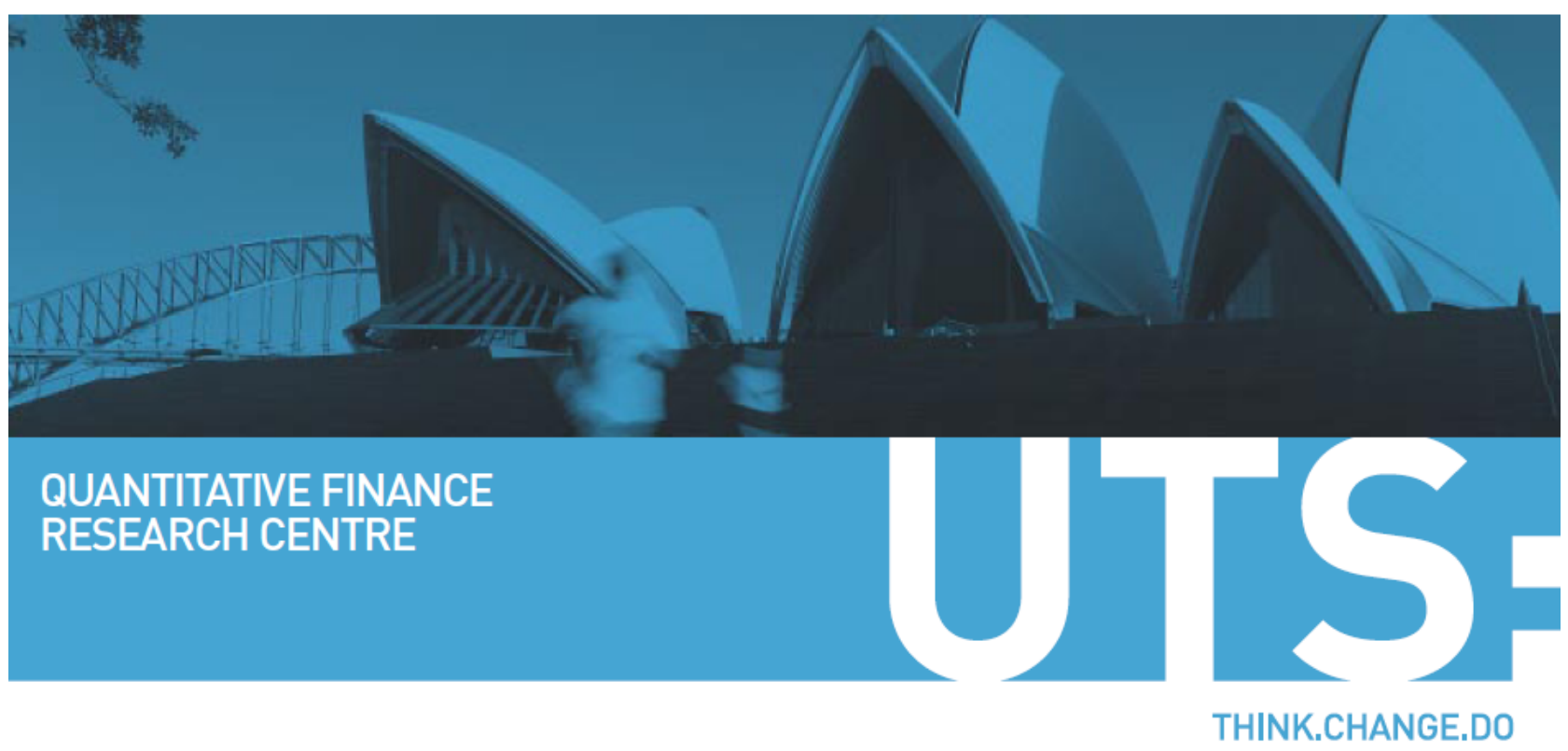

QUANTITATIVE FINANCE RESEARCH CENTRE

A Modern View on Merton’s Jump-Diffusion Model

Gerald H. L. Cheang and Carl Chiarella 


\title{
A Modern View on Merton's Jump-Diffusion Model
}

\author{
Gerald H. L. Cheang \\ Centre for Industrial and Applied Mathematics \\ School of Mathematics and Statistics \\ University of South Australia \\ GPO Box 2471, City West Campus, Adelaide \\ SA 5001, Australia \\ email: gerald.cheang@unisa.edu.au
}

\author{
Carl Chiarella \\ School of Finance and Economics \\ University of Technology, Sydney \\ PO Box 123, Broadway \\ NSW 2007, Australia \\ email: carl.chiarella@uts.edu.au
}

January 18, 2011

\begin{abstract}
Merton has provided a formula for the price of a European call option on a single stock where the stock price process contains a continuous Poisson jump component, in addition to a continuous log-normally distributed component. In Merton's analysis, the jump-risk is not priced. Thus the distribution of the jump-arrivals and the jump-sizes do not change under the change of measure. We go on to introduce a Radon-Nikodým derivative process that induces the change of measure from the market measure to an equivalent martingale measure. The choice of parameters in the Radon-Nikodým derivative allows us to price the option under different financial-economic scenarios. We introduce a hedging argument that eliminates the jump-risk in some sort of averaged sense, and derive an integro-partial differential equation of the option price that is related to the one obtained by Merton.
\end{abstract}

\section{KEY WORDS}

Financial derivatives, compound Poisson processes, equivalent martingale measure, hedging portfolio.

\section{Introduction}

Merton (1976) has provided a formula for the price of a European call option on a single stock where the stock price process contains a continuous Poisson jump component, in addition to a continuous log-normally distributed component. Merton's analysis in essence does not price the jump-risk. In this paper, we extend the results of Merton to the case where the market price of jump-risk is priced in the hedging portfolio. In Merton's case, financial economic arguments relating to systematic and unsystematic risk allows one to argue that the distribution of the Poisson jump components does not change under the change of measure. He considered a constant arrival intensity, log normally distributed jump sizes, set the market price of jump risk to zero and obtained a Poisson weighted sum of a Black-Scholes type formulae. He also considered the same hedge portfolio used by Black and Scholes (1973), namely one consisting of a position in the stock, the option and the risk-free asset only. In this case a perfect hedge does not exist and hedging was achieved by Merton by averaging out idiosyncratic risk. However, this leaves the market price of jump-risk unpriced, and also the distribution of the jump components remain unchanged. Further extensions to the Merton (1976) model include those by Anderson (1984) and Aase (1988). However these authors also make assumptions that amount to leaving the jump risk unpriced. Furthermore these later derivations do not appeal to the traditional hedging argument but rather appeal directly to the risk-neutral valuation principle and change of measure arguments.

However, the market which contains stocks with jump components is inherently incomplete in the Harrison and Pliska (1981) sense. When the market price of the jump-risk is accounted for, there are 
many equivalent martingale measures, and hence different prices for the option. For a single stock market, one could for example, apply a local risk-minimizing trading strategy in the manner of Schweizer (1991), Colwell and Elliott (1993), or a minimum entropy martingale measure approach in the manner of Miyahara (2001). Jeanblanc-Piqué and Pontier (1990) applied a general equilibrium model to the problem and used two assets driven by the same Wiener and Poisson noise factors. Jarrow and Madan (1995) included additional traded assets in order to hedge away the jump-risk in interest rate termstructure-related securities. Mercurio and Runggaldier (1993), and Runggaldier (2003), suggested that other assets driven by the same Wiener and Poisson noise factors as the stock be included in the hedge portfolio. In our case, we setup a hedging portfolio, in which two options of different maturities are required so that the jump-risk is properly priced in the portfolio. We use a hedging argument that eliminates the jump-risk by averaging over jumps. We then obtain the standard integro-partial differential equation for the option price and interpret economically the various parameters.

The paper develops as follows. In Section 2 we specify the asset pricing model. In Section 3 we introduce a Radon-Nikodým derivative that induces the change of measure from the market measure to an equivalent martingale measure for an option on the underlying asset. Section 4 then applies the results of Section 3 in the derivation of an integro-partial differential equation for the option price via the martingale approach. In section 5 we develop a hedging portfolio that is used in the derivation of the same integro-partial differential equation as in Section 4. The hedging portfolio also takes into account the market price of jump-risk. In Section 6, a general pricing formula is obtained for a European style call option and we recover Merton's (1976) call option formula as a special case. Section 7 concludes.

\section{Merton's Jump-Diffusion Model}

Throughout this paper, as in Merton (1976), we assume that $S_{t}$ is the price of a financial asset whose return dynamics are given by

$$
\frac{d S_{t}}{S_{t-}}=(\mu-\lambda \kappa) d t+\sigma d B_{t}+\left[e^{J}-1\right] d N_{t}
$$

where $\mu$ is the instantaneous expected return per unit time, and $\sigma$ is the instantaneous volatility per unit time. The stochastic process $B_{t}$ is a standard Wiener process under the market measure $\mathbb{P}$. The process $N_{t}$ is a Poisson process, independent of the jump-sizes $J$ and the Wiener process $B_{t}$, with arrival intensity $\lambda$ per unit time under the measure $\mathbb{P}$, so that its increments satisfy

$$
d N_{t}= \begin{cases}1 & \text { with probability } \lambda d t \\ 0 & \text { with probability } 1-\lambda d t\end{cases}
$$

The expected proportional jump size is

$$
\kappa \equiv \mathbb{E}_{\mathbb{P}}\left[e^{J}-1\right] .
$$

Jumps arriving at different times are assumed to be independent of each other. A filtered probability measure space $\left(\Omega, \mathcal{F},\left\{\mathcal{F}_{t}\right\}, \mathbb{P}\right)$ is assumed where the filtration $\left\{\mathcal{F}_{t}\right\}$ is the natural filtration generated by the Wiener process $B_{t}$ and the compound Poisson process $\sum_{n=1}^{N_{t}} J_{n}$. The moment generating function of all the jump-sizes is given by

$$
M_{\mathbb{P}, J}(u)=\mathbb{E}_{\mathbb{P}}\left[e^{u J}\right] .
$$

We also assume that the stock pays a continuous dividend yield at rate $q$. For simplicity, we assume that all the parameters in our model are constants, although the model can easily be extended to one with time-varying but non-stochastic parameters. 


\section{A Radon-Nikodým Derivative}

The stock price model (1) is driven by both a Wiener component $B_{t}$ and a compound Poisson process $\sum_{n=1}^{N_{t}} J_{n}$. We consider what happens to both the Wiener component and the jump component when we do a change of measure from $\mathbb{P}$ to another measure $\mathbb{Q}$. In the context of our model, the Radon-Nikodým derivative restricted to any time $t \leq T$ takes the form

$$
\left.\frac{d \mathbb{Q}}{d \mathbb{P}}\right|_{t}=\exp \left[-\theta B_{t}-\frac{\theta^{2}}{2} t+\sum_{n=1}^{N_{t}}\left(\gamma J_{n}+\nu\right)-\lambda \kappa^{\prime} t\right],
$$

where

$$
\kappa^{\prime} \equiv e^{\nu} M_{\mathbb{P}, J}(\gamma)-1=e^{\nu} \mathbb{E}_{\mathbb{P}}\left(e^{\gamma J}\right)-1 .
$$

It can easily be verified that (5) satisfies the properties of a Radon-Nikodým derivative and it is a martingale with respect to the measure $\mathbb{P}$. The form of the Radon-Nikodým derivative in (5) is a more compact representation and illustrates the Esscher transform nature of the representation. In our application, the form (5) allows us to manipulate the parameters to produce families of equivalent martingale measures in the stock price model. More general representations expressed in terms of Lévy measures, jump measures or compensated jump measures can be found Colwell and Elliott (1993), Cont and Tankov (2004) and in Runggaldier (2003).

Lemma 1. Let $\mathbb{P}$ and $\mathbb{Q}$ be equivalent measures. Consider a probability measure space $\left(\Omega, \mathcal{F},\left\{\mathcal{F}_{t}\right\}, \mathbb{P}\right)$ such that $\left\{\mathcal{F}_{t}\right\}$ is the natural filtration generated by a Wiener process $B_{t}$ and a compound Poisson process $\sum_{n=0}^{N_{t}} J_{n}$ and a Radon-Nikodým derivative given by (5) where $\gamma \in \mathbb{R}, \nu \in \mathbb{R}$ and $\kappa^{\prime}$ is given by (6). Then the Wiener process $B_{t}$ has drift $-\theta$ under the measure $\mathbb{Q}$ and the compound Poisson process $\sum_{n=0}^{N_{t}} J_{n}$ under the measure $\mathbb{Q}$ has a new intensity rate $\tilde{\lambda}=\lambda\left(1+\kappa^{\prime}\right)$ and a new distribution for the jump-sizes the moment generating function of which is given by

$$
M_{\mathbb{Q}, J}(u)=\frac{M_{\mathbb{P}, J}(\gamma+u)}{M_{\mathbb{P}, J}(\gamma)} .
$$

Proof: See e.g., Cont and Tankov (2004) or Runggaldier (2003). This is a special case of Theorem 2.5 in Runggaldier (2003).

Note that if the distribution of the jumps $J$ in the measure $\mathbb{P}$ comes from an exponential family, then the distribution of $J$ under the measure $\mathbb{Q}$ as given by the moment generating function in (7), also comes from the same exponential family but with different parameters, e.g. see Gerber and Shiu (1994). It can be observed that even if $\nu=0$, as long as $\gamma$ is non-zero, there will be a change in the jump-size distribution under the change of measure, and this in turn leads to a change in the intensity of the jump arrivals. On the other hand, if $\gamma$ is zero, but $\nu$ is non-zero, then there is a change in the intensity of the jump-arrivals only. However, if $\nu=-\ln M_{\mathbb{P}, J}(\gamma)$, then the Radon-Nikodým derivative (5) takes the form

$$
\left.\frac{d \mathbb{Q}}{d \mathbb{P}}\right|_{t}=e^{-\theta B_{t}-\frac{\theta^{2}}{2} t} \times \prod_{n=1}^{N_{t}} \frac{e^{\gamma J_{n}}}{M_{\mathbb{P}, J}(\gamma)} .
$$

In this case, the intensity of the jump-arrivals does not change under the change of measure.

In our context, the asset price dynamics contain both Wiener components and jump components given by (1). In the Radon-Nikodým derivative (5), the choice of the market prices of risk for the Wiener process, $\theta$, will be determined by the choice of the other parameters $\gamma$ and $\nu$, which determine the market prices of risk for the jump component. Lemma 1 indicates the new intensity rate $\tilde{\lambda}$ and the new distributions of the jump-sizes under the martingale measure $\mathbb{Q}$. The Wiener process $B_{t}$ now has drift $-\theta$ under the measure $\mathbb{Q}$. 


\section{An Integro-Partial Differential Equation for the Option Price}

Consider a European style option, e.g. a call option with payoff $X_{T}\left(S_{T}\right)=\left(S_{T}-K\right)^{+}$at maturity time $T$. As stated in Section 2, we shall assume that the underlying asset pays a continuous dividend at the rate $q$ per unit time, so that the yield process of the stock is $S_{t} e^{q t}$. We assume the presence of a money market account $e^{r t}$ where $r$ is the risk-free rate. We require that both the discounted stock yield process $\left\{\frac{S_{t} e^{q t}}{e^{r t}}\right\}$ and the discounted option price process $\left\{\frac{X_{t}\left(S_{t}\right)}{e^{r t}}\right\}$ to be martingales under an equivalent martingale measure $\mathbb{Q}$. In the presence of jumps, it is well-known that the martingale measure $\mathbb{Q}$ is not unique. Instead, the martingale measure $\mathbb{Q}$ can be determined by the choice of the parameters $\gamma$ and $\nu$ in the Radon-Nikodým derivative (5), with the market price of Wiener risk $\theta$ determined by the martingale condition after the choices of $\gamma$ and $\nu$ have been made.

The dynamics of the discounted yield process is given by

$$
\begin{gathered}
d\left(\frac{S_{t} e^{q t}}{e^{r t}}\right)=\left(\frac{S_{t-} e^{q t}}{e^{r t}}\right)[(\mu+q-r-\lambda \kappa) d t \\
\left.+\sigma d B_{t}+\left[e^{J}-1\right] d N_{t}\right] .
\end{gathered}
$$

After choosing the parameters $\gamma$ and $\nu$ in the Radon-Nikodyḿ derivative (5), the jump-arrival process $N_{t}$ is Poisson with arrival intensity $\tilde{\lambda}=\lambda\left(1+\kappa^{\prime}\right)$ and the distribution of the jump-sizes is given by the moment generating function

$$
M_{\mathbb{Q}, J}(u)=\frac{M_{\mathbb{P}, J}(u+\gamma)}{M_{\mathbb{P}, J}(\gamma)}
$$

from Lemma 1. It then follows that

$$
d\left(\frac{S_{t} e^{q t}}{e^{r t}}\right)=\left(\frac{S_{t-} e^{q t}}{e^{r t}}\right)\left[\sigma d \widetilde{B}_{t}-\tilde{\lambda} \tilde{\kappa} d t+\left[e^{J}-1\right] d N_{t}\right]
$$

where $\widetilde{B}_{t}$ is standard Brownian motion under the martingale measure $\mathbb{Q}$ and the expected jump-increment is $\tilde{\kappa}=\mathbb{E}_{\mathbb{Q}}\left[e^{J}\right]-1$. From Girsanov's Theorem,

$$
d \widetilde{B}_{t}=\theta d t+d B_{t}
$$

and the market price of Wiener risk $\theta$ satisfies the risk-premium equation

$$
\mu+q-r=\sigma \theta+\lambda \kappa-\tilde{\lambda} \tilde{\kappa} .
$$

In (11), the premium for Wiener risk is $\sigma \theta$, and the premium for jump-risk is $\lambda \kappa-\tilde{\lambda} \tilde{\kappa}$ which can be manipulated into the form

$$
\lambda \kappa\left[1-e^{\nu} \frac{M_{\mathbb{P}, J}(\gamma+1)-M_{\mathbb{P}, J}(\gamma)}{M_{\mathbb{P}, J}(1)-1}\right],
$$

in which the term

$$
1-e^{\nu} \frac{M_{\mathbb{P}, J}(\gamma+1)-M_{\mathbb{P}, J}(\gamma)}{M_{\mathbb{P}, J}(1)-1}
$$

can be interpreted as the market price of jump-risk. If the market price of jump-risk $\psi$ is specified, then the parameters $\gamma$ and $\nu$ in the Radon-Nikodým derivative (5) can be chosen such that

$$
1-e^{\nu} \frac{M_{\mathbb{P}, J}(\gamma+1)-M_{\mathbb{P}, J}(\gamma)}{M_{\mathbb{P}, J}(1)-1}=\psi .
$$


Through the removal of the discount factor in (10), the stock price dynamics (1) can also be expressed as

$$
\begin{aligned}
d S_{t}=S_{t-} & (r-q-\tilde{\lambda} \tilde{\kappa}) d t+\sigma S_{t-} d \widetilde{B}_{t} \\
+ & S_{t-}\left[e^{J}-1\right] d N_{t} .
\end{aligned}
$$

The following theorem gives the integro-partial differential equation for the option price.

Theorem 1. The European call option price satisfies the integro-partial differential equation

$$
\begin{aligned}
& \frac{\partial X_{t}\left(S_{t-}\right)}{\partial t}+S_{t-}(r-q-\tilde{\lambda} \tilde{\kappa}) \frac{\partial X_{t}\left(S_{t-}\right)}{\partial S} \\
& +\frac{1}{2} \sigma^{2} S_{t-}^{2} \frac{\partial^{2} X_{t}\left(S_{t-}\right)}{\partial S^{2}}+\tilde{\lambda} \mathbb{E}_{\mathbb{Q}}^{J}\left[X_{t}\left(S_{t-} e^{J}\right)-X_{t}\left(S_{t-}\right)\right] \\
& \quad=r X_{t}\left(S_{t-}\right)
\end{aligned}
$$

with terminal condition $X_{T}\left(S_{T}\right)=\left(S_{T}-K\right)^{+}$.

Proof. We require the discounted option price process $\left\{\frac{X_{t}\left(S_{t}\right)}{e^{r t}}\right\}$ to be a martingale under the martingale measure $\mathbb{Q}$. Since the option price $X_{t}\left(S_{t}\right)$ is a function of $S_{t}$, through the application of Itô's Lemma for jump-diffusion processes, the stochastic differential equation satisfied by this quantity is

$$
\begin{aligned}
d\left(\frac{X_{t}\left(S_{t}\right)}{e^{r t}}\right)= & {\left[e^{-r t} \frac{\partial X_{t}\left(S_{t-}\right)}{\partial t}-r \frac{X_{t}\left(S_{t-}\right)}{e^{r t}}\right.} \\
& +\frac{S_{t-}}{e^{r t}}(r-q-\tilde{\lambda} \tilde{\kappa}) \frac{\partial X_{t}\left(S_{t-}\right)}{\partial S} \\
& +\frac{1}{2} \sigma^{2} \frac{S_{t-}^{2}}{e^{r t}} \frac{\partial^{2} X_{t}\left(S_{t-}\right)}{\partial S^{2}} \\
& \left.+\frac{\tilde{\lambda}}{e^{r t}} \mathbb{E}_{\mathbb{Q}}^{J}\left[X_{t}\left(S_{t-} e^{J}\right)-X_{t}\left(S_{t-}\right)\right]\right] d t \\
& +\frac{\sigma S_{t-}}{e^{r t}} \frac{\partial X_{t}\left(S_{t-}\right)}{\partial S} d \widetilde{B}_{t} \\
& -\frac{\tilde{\lambda}}{e^{r t}} \mathbb{E}_{\mathbb{Q}}^{J}\left[X_{t}\left(S_{t-} e^{J}\right)-X_{t}\left(S_{t-}\right)\right] d t \\
& +\frac{1}{e^{r t}}\left[X_{t}\left(S_{t-} e^{J}\right)-X_{t}\left(S_{t-}\right)\right] d N_{t} .
\end{aligned}
$$

In (17), the coefficient of $d t$ must be zero in order that $\left\{\frac{X_{t}\left(S_{t}\right)}{e^{r t}}\right\}$ be a martingale under the martingale measure $\mathbb{Q}$, hence we obtain the integro-partial differential equation (16).

Using the fact that $\left\{\frac{X_{t}\left(S_{t}\right)}{e^{r t}}\right\}$ is a martingale under $\mathbb{Q}$, the solution for the option price in the form of a conditional expectation of the discounted final payoff can be written as

$$
X_{t}\left(S_{t}\right)=e^{r t} \mathbb{E}_{\mathbb{Q}}\left[\frac{X_{T}\left(S_{T}\right)}{e^{r T}} \mid \mathcal{F}_{t}\right] .
$$

\section{The Hedging Portfolio}

Now in order to obtain some economic intuition, we derive the integro-partial differential equation (16) by use of a hedging argument in some appropriate sense to be made clear below. Following Runggaldier (2003), where two options expiring at different maturities are needed to hedge an option where the underlying stock price return follows jump-diffusion dynamics, here our portfolio also consists of two 
similar options with differing maturities, that is, $X_{1, t}\left(S_{t}\right)$ and $X_{2, t}\left(S_{t}\right)$, expiring at arbitrary maturity dates $T_{1}$ and $T_{2}$ respectively. The value of the portfolio is

$$
\Pi_{t}=Q_{1} X_{1, t}\left(S_{t}\right)+Q_{2} X_{2, t}\left(S_{t}\right)+Q_{S} S_{t},
$$

where $Q_{1}$ and $Q_{2}$ are time-varying positions in the respective options, and $Q_{S}$ is a time-varying position in the stock. Adopting the short-form notation $X_{i, t}=X_{i, t}\left(S_{t}\right)(i=1,2)$ for the option price at time $t$ when the underlying stock price is $S_{t}$, and $X_{i, t-}=X_{i, t}\left(S_{t-}\right)$, the application of Itô's Lemma for jump-diffusion processes yields the dynamics for the option prices

$$
\begin{aligned}
\frac{d X_{i, t}}{X_{i, t-}}= & {\left[\mu_{X_{i}}-\lambda \kappa_{X_{i}}\right] d t+\sigma_{X_{i}} X_{i, t-} d B_{t} } \\
& +\left[e^{J_{X_{i}}}-1\right] d N_{t},
\end{aligned}
$$

where the drift of the option $X_{i}$ is denoted by

$$
\begin{aligned}
\mu_{X_{i}} X_{i, t}= & \frac{\partial X_{i, t-}}{\partial t}+\left(\mu_{i}-\lambda \kappa_{X_{i}}\right) S_{t-} \frac{\partial X_{i, t}}{\partial S} \\
& +\sigma^{2} \frac{S_{t-}^{2}}{2} \frac{\partial^{2} X_{i, t-}}{\partial S^{2}}+\lambda \kappa_{X_{i}} X_{i, t-},
\end{aligned}
$$

the option price volatility is denoted by

$$
\sigma_{X_{i}} X_{i, t-}=\sigma S_{t-} \frac{\partial X_{i, t-}}{\partial S}
$$

the expected jump-increment in the option price is

$$
X_{i, t-} \kappa_{X_{i}}=\mathbb{E}_{\mathbb{P}}^{J}\left[X_{i, t}\left(S_{t-} e^{J}\right)-X_{i, t-}\right],
$$

with the option price increment being given by

$$
\left[e^{J_{X_{i}}}-1\right] X_{i, t-}=X_{i, t}\left(S_{t-} e^{J}\right)-X_{i, t-} .
$$

The infinitesimal change in the portfolio value $d \Pi_{t}$ over a time interval $[t, t+d t)$ evolves according to

$$
d \Pi_{t}=Q_{1} d X_{1, t}+Q_{2} d X_{2, t}+Q_{S} d S_{t}+Q_{S} q S_{t-} d t .
$$

The change in the value of the portfolio $\Pi_{t}$ is thus given by

$$
\begin{aligned}
& d \Pi_{t}=\sum_{i=1}^{2}\left[\left(\mu_{X_{i}}-\lambda \kappa_{X_{i}}\right) d t+\sigma_{X_{i}} d B_{t}\right. \\
& \left.+\left[e^{J_{X_{i}}}-1\right] d N_{t}\right] Q_{i} X_{i, t-} \\
& +\left[(\mu+q-\lambda \kappa) d t+\sigma d B_{t}\right. \\
& \left.+\left[e^{J}-1\right] d N_{t}\right] Q_{S} S_{t-} \\
& =\left[Q_{S} S_{t-}(\mu+q-\lambda \kappa)+Q_{1} X_{1, t-}\right. \\
& \left.\left(\mu_{X_{1}}-\lambda \kappa_{X_{1}}\right)+Q_{2} X_{2, t-}\left(\mu_{X_{2}}-\lambda \kappa_{X_{2}}\right)\right] d t \\
& +\left[Q_{S} S_{t-} \sigma+Q_{1} X_{1, t-} \sigma_{X_{1}}\right. \\
& \left.+Q_{2} X_{2, t-} \sigma_{X_{2}}\right] d B_{t} \\
& +\left[Q_{S} S_{t-}\left[e^{J}-1\right]+Q_{1} X_{1, t-}\left[e^{J_{X_{1}}}-1\right]\right. \\
& +Q_{2} X_{2, t-}\left[e^{\left.J_{X_{2}}-1\right]}\right] d N_{t} \text {. }
\end{aligned}
$$


As first explained by Merton (1976), in a jump-diffusion model it is not possible to hedge away the idiosyncratic risks due to the jumps. Merton chose to leave the jump-risks unpriced, resulting in no change in the distribution of the jump components of his model under the change of measure. We choose an approach that considers a martingale measure under which the distribution of the jump components may change. Thus the market price of jump-risk remains present in our model. The choice of our equivalent martingale measure corresponds to a perfect hedge only when the jump-size $J$ takes on some particular value, say $J=J^{*}$, or if the jump-sizes have been averaged out with respect to some equivalent martingale measure so that some pre-determined value for the average relative jump-size increment is obtained.

We opt for the procedure of averaging out the jump-sizes according to some equivalent martingale measure $\hat{\mathbb{Q}}$ so that the portfolio (19) with the jumps averaged out under $\widehat{\mathbb{Q}}$ becomes

$$
\mathbb{E}_{\hat{\mathbb{Q}}}^{J} \Pi_{t}=\hat{Q}_{1} \mathbb{E}_{\hat{\mathbb{Q}}}^{J} X_{1, t}\left(S_{t}\right)+\hat{Q}_{2} \mathbb{E}_{\hat{\mathbb{Q}}}^{J} X_{2, t}\left(S_{t}\right)+\hat{Q}_{S} \mathbb{E}_{\hat{\mathbb{Q}}}^{J} S_{t},
$$

where $\mathbb{E}_{\hat{\mathbb{Q}}}^{J}$ denotes averaging the jumps under $\hat{\mathbb{Q}}$. The time-varying weights $\hat{Q}_{1}, \hat{Q}_{2}$ and $\hat{Q}_{3}$ will be the optimal weights over the interval $[t, t+d t)$ after the jumps have been averaged out. They correspond to a particular choice for the market price of jump risk to be discussed later. It will also be shown that these weights do not depend on the jump-sizes over the interval $[t, t+d t)$. Then by replacing the related quantities in (19) and (22) with their averaged out equivalent, we obtain

$$
\begin{aligned}
d\left(\mathbb{E}_{\hat{\mathbb{Q}}}^{J} \Pi_{t}\right)=[ & \hat{Q}_{S} S_{t-}(\mu+q-\lambda \kappa) \\
& \left.+\sum_{i=1}^{2} \hat{Q}_{i} X_{i, t-}\left(\mu_{X_{i}}-\lambda \kappa_{X_{i}}\right)\right] d t \\
+ & {\left[\hat{Q}_{S} S_{t-} \sigma+\hat{Q}_{1} X_{1, t-} \sigma_{X_{1}}\right.} \\
& \left.+\hat{Q}_{2} X_{2, t-} \sigma_{X_{2}}\right] d B_{t} \\
+ & {\left[\hat{Q}_{S} S_{t-} \hat{\kappa}+\hat{Q}_{1} X_{1, t-} \hat{\kappa}_{X_{1}}\right.} \\
& \left.+\hat{Q}_{2} X_{2, t-} \hat{\kappa}_{X_{2}}\right] d N_{t},
\end{aligned}
$$

where

$$
\begin{aligned}
& \hat{\kappa}=\mathbb{E}_{\hat{\mathbb{Q}}}^{J}\left[e^{J^{*}}-1\right], \\
& X_{i, t-} \hat{\kappa}_{X_{i}}=\mathbb{E}_{\hat{\mathbb{Q}}}^{J}\left[X_{i, t}\left(S_{t-} e^{J}\right)-X_{i, t-}\right], \\
& X_{t-} \hat{\kappa}_{X}=\mathbb{E}_{\hat{\mathbb{Q}}}^{J}\left[X_{t}\left(S_{t-} e^{J}\right)-X_{t-}\right] .
\end{aligned}
$$

We need to choose the weights in (24) to remove the Wiener risk and the jump-risk. Thus the condition for removing Wiener risk is

$$
\hat{Q}_{1} X_{1, t-} \sigma_{X_{1}}+\hat{Q}_{2} X_{2, t-} \sigma_{X_{2}}=-\hat{Q}_{S} S_{t-} \sigma
$$

and the condition for removing jump risk is

$$
\hat{Q}_{1} X_{1, t-} \hat{\kappa}_{X_{1}}+\hat{Q}_{2} X_{2, t-} \hat{\kappa}_{X_{2}}=-\hat{Q}_{S} S_{t-} \hat{\kappa} .
$$

Solving (25) and (26) for $Q_{1}, Q_{2}$ we obtain

$$
\hat{Q}_{1}=-\frac{\hat{Q}_{S} S_{t-}}{X_{1, t-}}\left(\frac{\sigma \hat{\kappa}_{X_{2}}-\sigma_{X_{2}} \hat{\kappa}}{\sigma_{X_{1}} \hat{\kappa}_{X_{2}}-\sigma_{X_{2}} \hat{\kappa}_{X_{1}}}\right)
$$


and

$$
\hat{Q}_{2}=\frac{\hat{Q}_{S} S_{t-}}{X_{2, t-}}\left(\frac{\sigma \hat{\kappa}_{X_{1}}-\sigma_{X_{1}} \hat{\kappa}}{\sigma_{X_{1}} \hat{\kappa}_{X_{2}}-\sigma_{X_{2}} \hat{\kappa}_{X_{1}}}\right) .
$$

Hence the portfolio with the jumps averaged out evolves according to

$$
\begin{aligned}
d\left(\mathbb{E}_{\hat{\mathbb{Q}}}^{J} \Pi_{t}\right)= & {\left[\hat{Q}_{S} S_{t-}(\mu+q-\lambda \kappa)\right.} \\
& \left.+\sum_{i=1}^{2} \hat{Q}_{i} X_{i, t-}\left(\mu_{X_{i}}-\lambda \kappa_{X_{i}}\right)\right] d t .
\end{aligned}
$$

We note that the weights were chosen to make $d\left(\mathbb{E}_{\hat{\mathbb{Q}}}^{J} \Pi_{t}\right)$ risk-less and hence the averaged portfolio (obtained by setting $Q_{1}=\hat{Q}_{1}, Q_{2}=\hat{Q}_{2}$ and $Q_{3}=\hat{Q}_{3}$ in (19)) has to grow at the risk-free rate to avoid arbitrage opportunities on average, so that

$$
d\left(\mathbb{E}_{\hat{\mathbb{Q}}}^{J} \Pi_{t}\right)=r\left[\hat{Q}_{S} S_{t-}+\hat{Q}_{1} X_{1, t-}+\hat{Q}_{2} X_{2, t-}\right] d t .
$$

Hence, equating the right hand sides of (29) and (30) we obtain

$$
\begin{aligned}
& \hat{Q}_{S} S_{t-}(\mu+q-\lambda \kappa-r) \\
& \quad+\sum_{i=1}^{2} \hat{Q}_{i} X_{i, t^{-}}\left(\mu_{X_{i}}-\lambda \kappa_{X_{i}}-r\right)=0 .
\end{aligned}
$$

Substituting for $\hat{Q}_{1}$ and $\hat{Q}_{2}$ in (27) and (28) into (31) we obtain

$$
\begin{aligned}
& \frac{\left(\mu_{X_{1}}-\lambda \kappa_{X_{1}}-r\right)-\frac{\sigma_{X_{1}}}{\sigma}(\mu+q-\lambda \kappa-r)}{\sigma \hat{\kappa}_{X_{1}}-\sigma_{X_{1}} \hat{\kappa}} \\
& \quad=\frac{\left(\mu_{X_{2}}-\lambda \kappa_{X_{2}}-r\right)-\frac{\sigma_{X_{2}}}{\sigma}(\mu+q-\lambda \kappa-r)}{\sigma \hat{\kappa}_{X_{2}}-\sigma_{X_{2}} \hat{\kappa}} .
\end{aligned}
$$

The left hand side and right hand side of (32) must be independent of the respective option's maturity time, so we can conclude that for any option $X_{t}$ based on the stock $S_{t}$, we must have

$$
\begin{aligned}
& \frac{\left(\mu_{X}-\lambda \kappa_{X}-r\right)-\frac{\sigma_{X}}{\sigma}(\mu+q-\lambda \kappa-r)}{\sigma \hat{\kappa}_{X}-\sigma_{X} \hat{\kappa}} \\
& =-\xi
\end{aligned}
$$

for some $\xi$ independent of maturity. Thus, by rearranging, we obtain

$$
\begin{aligned}
& \frac{\left(\mu_{X}-r-\lambda \kappa_{X}+\hat{\kappa}_{X} \sigma \xi\right)}{\sigma_{X}} \\
& =\frac{(\mu+q-r-\lambda \kappa+\hat{\kappa} \sigma \xi)}{\sigma} .
\end{aligned}
$$

We have let $\xi>0$ in (33) in order to ensure the correct sign for the market premium for the jumprisk in (34). Of course mathematically there is no reason to guarantee that the RHS of (33) should be negative. It is in fact an empirical issue as to whether the risk premium for bearing jump-risk is positive. Whilst there are a lot of empirical results in this area supporting the positivity of the ex-ante risk premium (e.g. Fama and French (2002)), we should also point to the studies of Boudoukh et al. (1993) and Walsh (2006) who report that the ex-ante market risk premium can on occasions be negative. 
Now recall the form of the Radon-Nikodým derivative (5). For (34) to make economic sense, the LHS has to be the risk premium less the jump-risk per unit volatility for the option, and the RHS, the risk premium less the jump-risk per unit volatility for the stock. After the jump-risk is removed from both sides of (34), then either expression is the market price of the Wiener risk $\theta$ in the Radon-Nikodým derivative (5). Hence it follows that the new jump intensity under $\widehat{\mathbb{Q}}$ has to be $\hat{\lambda}=\sigma \xi$.

In order to solve for the parameters $\nu$ and $\gamma$ in the Radon-Nikodým derivative (5), we need to solve simultaneously

$$
1+\hat{\kappa}=\frac{\mathbb{E}_{\mathbb{P}}\left[e^{(\gamma+1) J}\right]}{\mathbb{E}_{\mathbb{P}} e^{\gamma J}}
$$

and

$$
1+\hat{\kappa}=\frac{e^{\nu} \mathbb{E}_{\mathbb{P}}\left[e^{(\gamma+1) J}\right]}{\hat{\lambda}} .
$$

The values of the parameters $\hat{\nu}$ and $\hat{\gamma}$ from (35) and (36) yield the Radon-Nikodým derivative $\left.\frac{d \hat{\mathbb{Q}}}{d \mathbb{P}}\right|_{T}$. Thus the risk premium of the option in (34) with the jumps averaged out in the market measure $\mathbb{P}$ is

$$
\begin{aligned}
& \frac{\left(\mu_{X}-r-\lambda \kappa_{X}+\hat{\lambda} \mathbb{E}_{\mathbb{P}}^{J}\left[\left.\frac{d \hat{\mathbb{Q}}}{d \mathbb{P}}\right|_{T}\left[e^{J_{X}}-1\right]\right]\right)}{\sigma_{X}} \\
& =\frac{\left(\mu+q-r-\lambda \kappa+\hat{\lambda} \mathbb{E}_{\mathbb{P}}^{J}\left[\left.\frac{d \hat{\mathbb{Q}}}{d \mathbb{P}}\right|_{T}\left(e^{J}-1\right)\right]\right)}{\sigma},
\end{aligned}
$$

which is equivalent to

$$
\begin{aligned}
& \frac{\left(\mu_{X}-r-\lambda \kappa_{X}+\hat{\lambda} \mathbb{E}_{\hat{\mathbb{Q}}}^{J}\left[e^{J_{X}}-1\right]\right)}{\sigma_{X}} \\
& =\frac{\left(\mu+q-r-\lambda \kappa+\hat{\lambda} \mathbb{E}_{\hat{\mathbb{Q}}}^{J}\left[e^{J}-1\right]\right)}{\sigma},
\end{aligned}
$$

after expressing the expectations under $\mathbb{P}$ in (37) as expectations under the martingale measure $\hat{\mathbb{Q}}$ in (38).

Recall that $\hat{\kappa}$ is the expected jump-increment of the stock, and $\hat{\kappa}_{X}$ is the expected jump-increment in the option price, both in the equivalent martingale measure $\hat{\mathbb{Q}}$. Then the risk premium of the option from (38) satisfies

$$
\mu_{X}-r-\lambda \kappa_{X}+\hat{\lambda} \hat{\kappa}_{X}=\frac{\sigma_{X}}{\sigma}(\mu+q-r-\lambda \kappa+\hat{\lambda} \hat{\kappa}) .
$$

From the expression $\theta$ for the market price of Wiener risk (11), we see that (39) expresses the risk premium of the option less the jump-risk as the market price of Wiener risk $\theta$ scaled up by the option price volatility $\sigma_{X}$, so that (39) can be rewritten as

$$
\mu_{X}-r-\left(\lambda \kappa_{X}-\hat{\lambda} \hat{\kappa}_{X}\right)=\sigma_{X} \theta
$$

Finally we multiply both sides of (39) by $X_{t-}$ and substitute into the left side of (39) for

$$
\begin{aligned}
X_{t-} \mu_{X}= & \frac{\partial X_{t-}}{\partial t}+(\mu-\lambda \kappa) S_{t-} \frac{\partial X_{t-}}{\partial S} \\
& +\sigma^{2} \frac{S_{t-}^{2}}{2} \frac{\partial^{2} X_{t-}}{\partial S^{2}}+\lambda \kappa_{X} X_{t-}, \\
\sigma_{X} X_{t-}= & \sigma S_{t-} \frac{\partial X_{t-}}{\partial S}, \\
X_{t-} \kappa_{X}= & \mathbb{E}_{\mathbb{P}}^{J}\left[X_{t}\left(S_{t-} e^{J}\right)-X_{t-}\right]
\end{aligned}
$$


and

$$
X_{t-\hat{\kappa}_{X}}=\mathbb{E}_{\hat{\mathbb{Q}}}^{J}\left[X_{t}\left(S_{t-} e^{J}\right)-X_{t-}\right]
$$

Thus (39) becomes

$$
\begin{aligned}
\frac{\partial X_{t-}}{\partial t} & +(r-q-\hat{\lambda} \hat{\kappa}) S_{t-} \frac{\partial X_{t-}}{\partial S}+\sigma^{2} \frac{S_{t-}^{2}}{2} \frac{\partial^{2} X_{t-}}{\partial S^{2}} \\
& +\hat{\lambda} \mathbb{E}_{\hat{\mathbb{Q}}}^{J}\left[X_{t}\left(S_{t-} e^{J}\right)-X_{t-}\right]=r X_{t-},
\end{aligned}
$$

which is again the integro-partial differential equation (16), where the expectations are taken under the equivalent martingale measure $\hat{\mathbb{Q}}$. Note that if the values $\hat{\nu}$ and $\hat{\gamma}$ from (35) and (36) were used as the values of $\nu$ and $\gamma$ in the Radon-Nikodým derivative in Lemma 1, and applied to the martingale approach for the derivation of the integro-partial differential equation (16) in Section 4, then the martingale measure $\hat{\mathbb{Q}}$ is the same as $\mathbb{Q}$.

\section{Pricing the Call Option}

Let us now price the call option at time $t=0$. Following Merton (1976), we now assume that the jump-sizes are normally distributed with mean $\alpha$ and variance $\delta^{2}$ under the market measure $\mathbb{P}$. For a chosen $\gamma$ and $\nu$ in the Radon-Nikodým derivative (5), we see from the application of Lemma 1 that the jump-sizes will be normally distributed with mean $\tilde{\alpha}=\alpha+\gamma \delta^{2}$ with the same variance $\delta^{2}$, and the new intensity of the jump-arrivals is $\tilde{\lambda}=\lambda\left(1+\kappa^{\prime}\right)$, under the equivalent martingale measure $\mathbb{Q}$.

The next theorem gives the European call option price.

Theorem 2. Suppose the stock price follows the dynamics (1), and the stock pays a continuous dividend at the rate $q$. The European call option price $X_{0}\left(S_{0}\right)$ at time $t=0$ is given by

$$
\begin{aligned}
X_{0}\left(S_{0}\right)= & \sum_{n=0}^{\infty} e^{-\hat{\lambda} T} \frac{(\hat{\lambda} T)^{n}}{n !} \\
& \times\left[S_{0} e^{-q T} \Phi\left(d_{1, n}\right)-K e^{-r_{n} T} \Phi\left(d_{2, n}\right)\right],
\end{aligned}
$$

where

$$
d_{1, n}=\frac{\ln \frac{S_{0}}{K}+\left(r_{n}-q+\frac{\sigma_{n}^{2}}{2}\right) T}{\sigma_{n} \sqrt{T}}
$$

and

$$
d_{2, n}=d_{1, n}-\sigma_{n} \sqrt{T}
$$

with

$$
\begin{aligned}
\tilde{\alpha} & \equiv \alpha+\gamma \delta^{2} \\
\tilde{\lambda} & \equiv \lambda \exp \left[\nu+\gamma \alpha+\frac{\gamma^{2} \delta^{2}}{2}\right], \\
\tilde{\kappa} & \equiv \exp \left[\tilde{\alpha}+\frac{\delta^{2}}{2}\right]-1 \\
\hat{\lambda} & \equiv \lambda e^{\nu+\gamma^{2} \alpha+\frac{\gamma^{2} \delta^{2}}{2}} \times e^{\tilde{\alpha}+\frac{\delta^{2}}{2}} \\
r_{n} & \equiv r-\tilde{\lambda} \tilde{k}+\frac{n \tilde{\alpha}}{T}+\frac{n \delta^{2}}{2 T}
\end{aligned}
$$

and

$$
\sigma_{n}^{2} \equiv \sigma^{2}+\frac{n \delta^{2}}{T}
$$


Proof. Note that the option price discounted by the money market account $e^{r t}$ is a martingale in the martingale measure $\mathbb{Q}$. Let $A=\left\{S_{T}>K\right\}$ be the event that the option is in the money at maturity. The event $A$ is equivalent to the event that

$$
\left\{\sigma \widetilde{B}_{T}+\sum_{n=1}^{N_{T}} J_{n}>\ln \frac{S_{0}}{K}-\left(r-q-\tilde{\lambda} \tilde{\kappa}-\frac{\sigma^{2}}{2}\right) T\right\} .
$$

Hence from (18), the call option price is

$$
\begin{aligned}
X_{0}\left(S_{0}\right)= & e^{-r T} \mathbb{E}_{\mathbb{Q}}\left[\left(S_{T}-K\right) \mathbb{1}_{A}\right] \\
= & S_{0} e^{-q T} \\
& \quad \times \mathbb{E}_{\mathbb{Q}}\left[e^{-\frac{1}{2} \sigma^{2} T+\sigma \widetilde{B}_{T}-\tilde{\lambda} \tilde{\kappa} T+\sum_{n=1}^{N_{T}} J_{n}} \mathbb{1}_{A}\right] \\
& \quad-K e^{-r T} \mathbb{E}_{\mathbb{Q}} \mathbb{1}_{A} \\
= & S_{0} e^{-q T} \widetilde{\mathbb{Q}}(A)-K e^{-r T} \mathbb{Q}(A)
\end{aligned}
$$

where in (42), the Radon-Nikodým derivative is

$$
\left.\frac{d \widetilde{\mathbb{Q}}}{d \mathbb{Q}}\right|_{T}=e^{-\frac{1}{2} \sigma^{2} T+\sigma \widetilde{B}_{T}-\tilde{\lambda} \tilde{\kappa} T+\sum_{n=1}^{N_{T}} J_{n}}
$$

We note that

$$
\mathbb{Q}(A)=\sum_{n=0}^{\infty} e^{-\tilde{\lambda} T} \frac{(\tilde{\lambda} T)^{n}}{n !} \times \Phi\left(d_{2, n}\right)
$$

where

$$
d_{2, n}=\frac{\ln \frac{S_{0}}{K}+\left(r-q-\tilde{\lambda} \tilde{\kappa}-\frac{\sigma^{2}}{2}\right) T+n\left(\alpha+\gamma \delta^{2}\right)}{\sqrt{\left(\sigma^{2}+\frac{n \delta^{2}}{T}\right) T}} .
$$

Under the measure $\widetilde{\mathbb{Q}}$ and the application of Lemma 1, the Wiener component $\sigma \widetilde{B}_{T}$ is normally distributed as $N\left(\sigma^{2} T, \sigma^{2} T\right)$ and $J$ is normally distributed as $N\left(\alpha+\gamma \delta^{2}+\delta^{2}, \delta^{2}\right)$ and the Poisson process $N_{t}$ has intensity $\hat{\lambda}=\tilde{\lambda}(1+\tilde{\kappa})$. Hence

$$
\widetilde{\mathbb{Q}}(A)=\sum_{n=0}^{\infty} e^{-\hat{\lambda} T} \frac{(\hat{\lambda} T)^{n}}{n !} \times \Phi\left(d_{1, n}\right)
$$

where

$$
d_{1, n}=\frac{\ln \frac{S_{0}}{K}+\left(r-q-\tilde{\lambda} \tilde{\kappa}+\frac{\sigma^{2}}{2}\right) T+n\left(\alpha+\gamma \delta^{2}+\delta^{2}\right)}{\sqrt{\left(\sigma^{2}+\frac{n \delta^{2}}{T}\right) T}} .
$$

Remark. In the proof of Theorem 1, the decomposition of the option price in (42) is analogous to that obtained by Geman et al. (1995) for the pure-diffusion case. The measure $\widetilde{\mathbb{Q}}$ corresponds to the equivalent martingale measure with the stock price as the numéraire.

We conclude this section by noting that when the parameters $\gamma$ and $\nu$ are set to zero in the RadonNikodým derivative (5), we recover Merton's (1976) model as a special case. In this case since the parameters $\gamma$ and $\nu$ are set to zero, the market price of jump-risk in (11) to (13) is not priced. 


\section{Conclusion}

This paper has extended the analysis of Merton (1976) to the case where the distribution of the jumparrivals and the jump-sizes change under the change of measure. A Radon-Nikodým derivative process that induces the change of measure through the choice of suitable parameters has been introduced. We have shown how the non-uniqueness of the option price manifests itself through variations in the parameters of the Radon-Nikodým derivative that induces the change of measure. Through a hedging portfolio that averages over the jumps, we relate the change of the distributions of the jump components to the market price of jump-risk. We also derive the standard pricing integro-partial differential equation.

\section{Acknowledgement}

The authors acknowledge the helpful discussions with Wolfgang Runggaldier over an earlier draft of a multi-asset version of this paper. The usual caveat applies.

\section{References}

[1] K.K. Aase, Contingent claim valuation when the security price is a combination of an Itô process and a random point process, Stochatic Processes and Applications, 28, 1988, 185-220.

[2] W.J. Anderson, Hedge portfolios and the Black-Scholes equations, Stochastic Analysis and Applications, 2(1), 1984, 1-11.

[3] F. Black and M. Scholes, The pricing of corporate liabilities, Journal of Political Economy, 81, 1973, 637-659

[4] J. Boudoukh, M. Richardson and T. Smith, Is the ex-ante risk premium always positive? A new approach to testing conditional asset pricing models, Journal of Financial Economics, 34, 1993, 387-408.

[5] D.B. Colwell and R.J. Elliott, Discontinuous asset prices and non-attainable contingent claims, Mathematical Finance, 3(3), 1993, 295-308.

[6] R. Cont and P. Tankov. Financial modelling with jump processes (London: Chapman and Hall/CRC, 2004).

[7] E.F. Fama and K.R. French, The equity premium, Journal of Finance, 57(2), 2002, 637-655.

[8] H. Geman, N. El-Karoui and J.-C. Rochet, Change of numéraire, changes of probability measure and option pricing, Journal of Applied Probability, 32(2), 1995, 443-459.

[9] H.U. Gerber and E.S.W. Shiu, Option pricing by Esscher transforms, Trans., Society of Actuaries, 46, 1994, 99-191.

[10] J.M. Harrison and S.R. Pliska, Martingales and stochastic integrals in the theory of continous trading, Stoch. Process. Appl., 11(3), 1981, 215-260.

[11] R. Jarrow and D.B. Madan, Option pricing using the term structure of interest rates to hedge systematic discontinuities in asset returns, Mathematical Finance, 5(4), 1995, 311-356.

[12] M. Jeanblanc-Picqué and M. Pontier, Optimal portfolio for a small investor in a market model with discontinuous prices, Applied Mathematical Optimization, 22, 1990, 287-310.

[13] F. Mercurio and W.J. Runggaldier, Option pricing for jump diffusions: approximations and their interpretation, Mathematical Finance, 3(2), 1993, 191-200.

[14] R.C. Merton, Option pricing when underlying stock returns are discontinuous, Journal of Financial Economics, 3(1), 1976, 125-144.

[15] W.J. Runggaldier, Jump diffusion models, in S.T. Rachev (Ed.) Handbook of heavy tailed distributions in finance, (North-Holland: Elsevier, 2003) 169-209. 
[16] M. Schweizer, Option hedging for semimartingales, Stochastic Processes and Applications, 37, 1991, 339-363.

[17] K.D. Walsh, Is the ex-ante risk premium always positive? Further evidence, Australian Journal of Management, 31(1), 2006, 93-113. 